Typeset with jpsj2.cls $<$ ver.1.2 $>$

LETTER

\title{
Impurity Effect as a Probe for the Gap Function in the Filled Skutterudite Compound Superconductor $\mathrm{PrOs}_{4} \mathrm{Sb}_{12}$ : Sb-NQR Study
}

\author{
Masahide NishiYama, Takayuki Kato, Hitoshi Sugawara ${ }^{1}$, Daisuke KIKuCHI ${ }^{2}$, Hideyuki \\ SATO $^{2}$, Hisatomo HARIMA ${ }^{3}$, and Guo-qing ZhenG \\ Department of Physics, Okayama University, Okayama 700-8530, Japan \\ ${ }^{1}$ Department of Mathematical and Natural Sciences, Faculty of Integrated Arts and Sciences, The \\ University of Tokushima, Tokushima 770-8502, Japan \\ ${ }^{2}$ Department of Physics, Graduate School of Science, Tokyo Metropolitan University, Hachioji \\ 192-0397, Japan \\ ${ }^{3}$ Department of Physics, Kobe University, Kobe 657-8501, Japan
}

\begin{abstract}
We have carried out nuclear quadrupole resonance (NQR) measurements in the filled skutterudite compounds $\operatorname{Pr}\left(\mathrm{Os}_{1-x} \mathrm{Ru}_{x}\right)_{4} \mathrm{Sb}_{12}(x=0.1,0.2)$, in order to gain insights into the symmetry of the superconducting gap function. Upon replacing Os with $\mathrm{Ru}$, the spin-lattice relaxation rate $1 / T_{1}$ becomes proportional to temperature $(T)$ at low $T$ far below $T_{c}$, and the magnitude of $\left(1 / T_{1} T\right)_{\text {low-T }}$ increases with increasing $\mathrm{Ru}$ content. These results indicate that a finite density of states is induced at the Fermi level by the impurity, and thus suggest that there exist nodes in the gap function of $\mathrm{PrOs}_{4} \mathrm{Sb}_{12}$.
\end{abstract}

KEYWORDS: filled skutterudite, Heavy fermion, superconductivity, impurity effect, nuclear quadrupole resonance, residual DOS

The superconductivity at $T_{c}=1.85 \mathrm{~K}$ discovered in the filled skutterudite compound $\mathrm{PrOs}_{4} \mathrm{Sb}_{12}$ has attracted much attention. ${ }^{1,2}$ This is the first Pr-based heavy fermion superconductor, in which the heavy mass has been suggested by the large specific heat jump $\Delta C / T_{\mathrm{c}} \sim 500 \mathrm{~mJ} / \mathrm{K}^{2} \cdot \mathrm{mol}$ at $T_{\mathrm{c}}{ }^{1,2}$ and directly confirmed by de Haas-van Alphen (dHvA) experiments. ${ }^{3}$ The crystal electric field (CEF) ground state is a $\Gamma_{1}$ singlet, which is separated by the first excited state of the $\Gamma_{4}^{(2)}$ triplet by a gap of $\Delta_{C E F}=10 \mathrm{~K} .{ }^{4-9}$ Because of this small $\Delta_{C E F}$, the role of quadrupole moment fluctuations arising from the $\Gamma_{4}^{(2)}$ state has attracted much attention. It has been speculated that the interaction between the quadrupolar moments and the conduction-electron charges may be responsible for the heavy mass, thus representing a charge-scattering version of the Kondo effect. ${ }^{10}$ The contrasting behavior that the isostructural compound $\mathrm{PrRu}_{4} \mathrm{Sb}_{12}$ with $\Delta_{C E F}=70 \mathrm{~K}$ has a much smaller $m^{* 11,12}$ has increased such expectation.

One of the first steps toward understanding the Pr-based heavy fermions may be the determination of the gap symmetry of the superconductivity. Previous nuclear quadrupole resonance (NQR) measurements have revealed the unconventional nature of the supercon- 
ductivity. ${ }^{13}$ No coherence peak was found in the spin-lattice relaxation rate $1 / T_{1}$ just below $T_{c}$, which suggests non-BCS-type superconductivity and is in contrast to the $s$-wave bahavior subsequently observed in $\mathrm{PrRu}_{4} \mathrm{Sb}_{12} \cdot{ }^{14}$ At low temperatures, $1 / T_{1}$ does not follow a powerlaw $T$-dependence, which is in contrast to the observation of a $T^{3}$ variation in other known heavy fermion superconductors. ${ }^{15}$ Together with the exponential decrease in the penetration depth found by muon spin relaxation, ${ }^{16}$ it has been suggested that the superconducting gap is isotropic. However, oscillations with respect to the magnetic field angle have been found in angle-resolved thermal conductivity measurements, which suggests that the gap is anisotropic. $^{17}$

In this study, we use an impurity as a probe for the gap function. We replace Os with $\mathrm{Ru}$, and study its effect on the superconducting density of states (DOS) using the NQR technique. It has been known for some time that a non-magnetic impurity is a powerful "smoking gun" for the gap function. In $s$-wave superconductors, a paramagnetic impurity produces a bound state within the gap (Yu-Shiba state), ${ }^{18,19}$ and non-magnetic impurities smear out the gap anisotropy, if any. ${ }^{20}$ In contrast, if there are nodes in the gap function, a finite DOS is brought about at the Fermi level, ${ }^{21,22}$ which can be detected experimentally. ${ }^{15}$

Single crystals of $\operatorname{Pr}\left(\mathrm{Os}_{1-x} \mathrm{Ru}_{x}\right)_{4} \mathrm{Sb}_{12},(x=0.1$ and 0.2$)$ were grown by the Sb-flux method. $T_{c}$ 's are $1.6 \mathrm{~K}$ and $1.4 \mathrm{~K}$ for $x=0.1$ and 0.2 , respectively, in agreement with those previously reported. ${ }^{23,24}$ For NQR measurements, the single crystals were powdered in order to allow the radio-frequency magnetic field to penetrate into the sample. The NQR measurements were performed by using a phase coherent spectrometer. The spin-lattice relaxation rate, $1 / T_{1}$, for ${ }^{123} \mathrm{Sb}$ were measured using the saturation-recovery method. Data below $1.4 \mathrm{~K}$ were collected using a ${ }^{3} \mathrm{He} /{ }^{4} \mathrm{He}$ dilution refrigerator.

Figure 1 shows the NQR spectra for the $x=0.1$ and 0.2 samples. For comparison, the data for the two end-member compounds ${ }^{13,14}$ are also shown in Figs 1 (a) and 1(d). In the Ru-doped samples, in addition to the spectrum peaks that are located at the same positions as those for pure $\mathrm{PrOs}_{4} \mathrm{Sb}_{12}$, there appear new sets of peaks that are characterized by $\nu_{Q}=26$ $\mathrm{MHz}$ and the asymmetry parameter $\eta=0.43$ for ${ }^{123} \mathrm{Sb}$. Here, $\nu_{Q}$ and $\eta$ are defined as $\nu_{Q} \equiv$ $\nu_{z}=\frac{3}{2 I(2 I-1) h} e^{2} Q \frac{\partial^{2} V}{\partial z^{2}}$ and $\eta=\left|\nu_{x}-\nu_{y}\right| / \nu_{z}$ with $Q$ being the nuclear quadrupolar moment and $\frac{\partial^{2} V}{\partial \alpha^{2}}(\alpha=x, y, z)$ being the electric field gradient at the position of the nucleus. ${ }^{25}$ It should be emphasized that these peaks (labelled as "peaks 2" in Table I) are different from those for pure $\mathrm{PrRu}_{4} \mathrm{Sb}_{12}$. These new peaks are due to the alloying effect, which indicates that the alloying is homogenous and there is no phase separation. Such a feature has been seen before in other heavy fermion compounds. For example, in Ir-doped CeRhIn 5 , upon replacing $\mathrm{Rh}$ with Ir, there emerges a new set of NQR peaks that is different from that for $\mathrm{CeRhIn}_{5}$ or CeIrIn ${ }_{5} \cdot{ }^{26}$ Nonetheless, $1 / T_{1}$ measured at the new set of peaks shows the same $T$-dependence as that measured at those corresponding to $\mathrm{CeRhIn}_{5}$ or $\mathrm{CeIrIn} 5$, indicating that the new set 


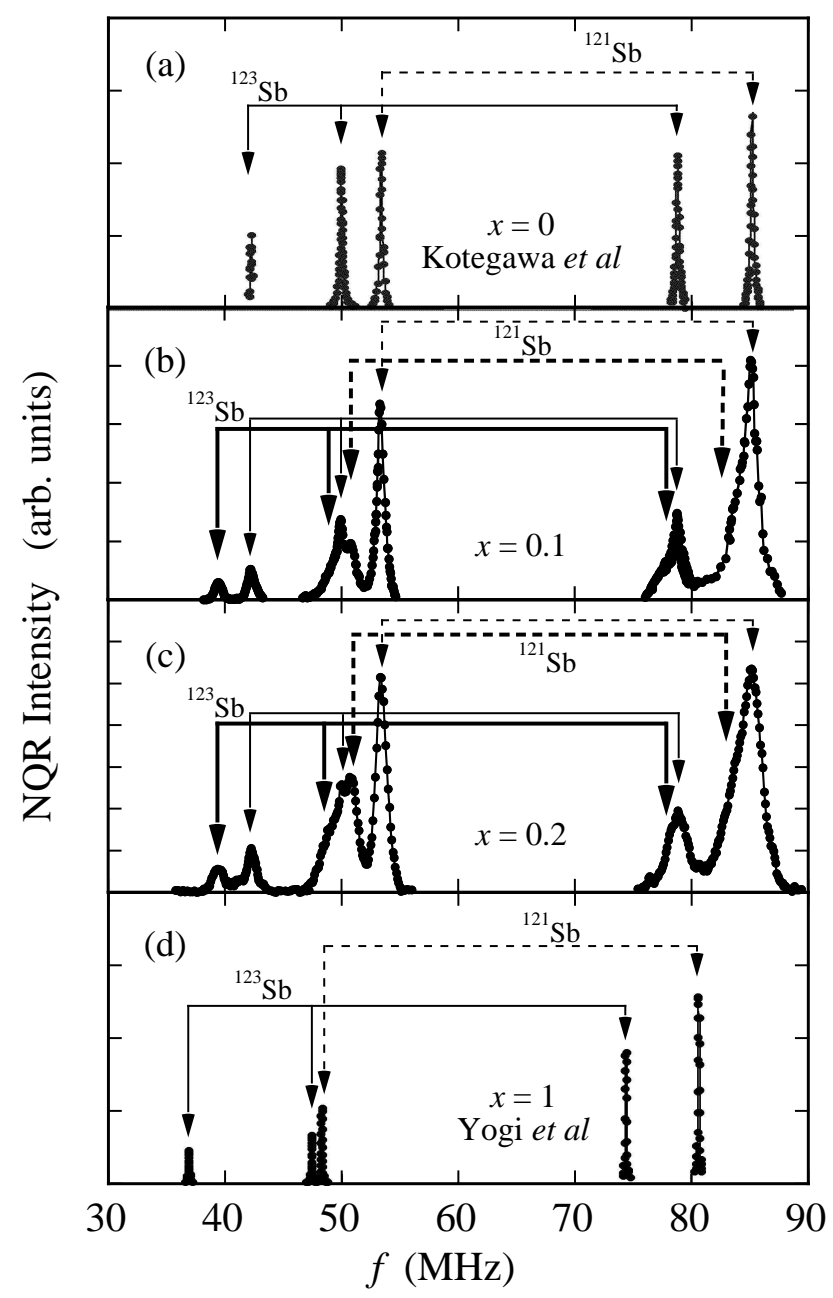

Fig. 1. Sb-NQR spectra of $\operatorname{Pr}\left(\mathrm{Os}_{1-x} \mathrm{Ru}_{x}\right)_{4} \mathrm{Sb}_{12}$ at $4.2 \mathrm{~K}$. The data for $x=0$ and $x=1$ are from Kotegawa et al. ${ }^{13}$ and Yogi et al. ${ }^{14}$ In (b) and (c), the spectra indicated by the thin solid lines (broken lines) correspond to ${ }^{123} \mathrm{Sb}\left({ }^{121} \mathrm{Sb}\right)$ signals seen in $\mathrm{PrOs}_{4} \mathrm{Sb}_{12}$, while the spectra indicated by the thick markers are the new sets arising from $\mathrm{Ru}$ doping.

of peaks is not due to phase-separated regions but rather to the homogenous alloying.

The nuclear spin-lattice relaxation rate $1 / T_{1}$ was measured at the peaks at $49.9 \mathrm{MHz}$ $( \pm 3 / 2 \longleftrightarrow \rightarrow \pm 5 / 2$ transition $)$ and $42.2 \mathrm{MHz}( \pm 1 / 2 \leftarrow \rightarrow \pm 3 / 2$ transition). Both measurements yield the same results. Even for $x=0.2$, no influence on $T_{1}$ due to the overlapping of the ${ }^{121} \mathrm{Sb}$ line was seen in the measurements at the $49.9 \mathrm{MHz}$ peak, probably because the $H_{1}$ we used for observing the echo is much smaller than the frequency difference between the ${ }^{121} \mathrm{Sb}$ and ${ }^{123} \mathrm{Sb}$ peaks, which is about $0.8 \mathrm{MHz}$, and the ${ }^{121} \mathrm{Sb}$ peak has a small full width at half maximum (FWHM) of $0.5 \mathrm{MHz}$. Above $T \sim 100 \mathrm{~K}$, the nuclear magnetization can be fitted excellently using the expected theoretical rate equation. ${ }^{27}$ However, below $T \sim 100 \mathrm{~K}$, it cannot be fitted using the theoretical curve with a single component of $T_{1}$. This is also true at the peak at 42.2 
Table I. NQR frequency $\nu_{Q}$ and asymmetric parameter $\eta$ for ${ }^{123} \mathrm{Sb}$ in $\operatorname{Pr}\left(\mathrm{Os}_{1-x} \mathrm{Ru}_{x}\right)_{4} \mathrm{Sb}_{12}$. Also shown are the CEF gap $\Delta_{\mathrm{CEF}}$ and the heavy quasiparticle enhancement factor $\beta$ (see text).

\begin{tabular}{|c||cc|cc||cc|}
\hline \multicolumn{1}{|c||}{} & \multicolumn{2}{c|}{ Peaks 1} & \multicolumn{2}{c||}{ Peaks 2 } & & \\
\cline { 2 - 5 }$x$ & $\nu_{Q}(\mathrm{MHz})$ & $\eta$ & $\nu_{Q}(\mathrm{MHz})$ & $\eta$ & $\beta$ & $\Delta_{\mathrm{CEF}}(\mathrm{K})$ \\
\hline 0 & 26.8 & 0.41 & - & - & 26.7 & 8 \\
0.1 & 26.8 & 0.41 & 26 & 0.43 & 16 & 10 \\
0.2 & 26.8 & 0.41 & 26 & 0.43 & 11 & 13 \\
1 & 25.2 & 0.46 & - & - & - & - \\
\hline
\end{tabular}

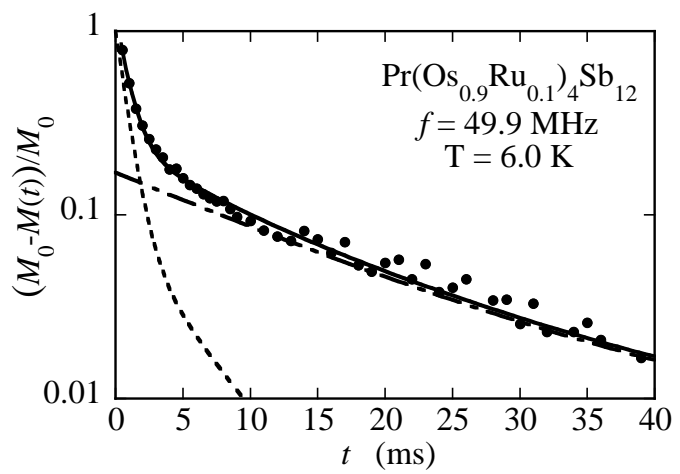

Fig. 2. Nuclear magnetization recovery curve. The broken and dot-dashed curves indicate the recovery curves due to the fast and slow relaxation components, respectively. The solid curve indicates the sum of the two components.

$\mathrm{MHz}$ that is free from overlapping by other transition lines. Figure 2 shows the decay curve of the nuclear magnetization at $T=6 \mathrm{~K}$. As will become clearer later, the inhomogeneous $T_{1}$ is an intrinsic property of the alloyed samples. The one-component behavior for $T \geq 100 \mathrm{~K}$ is rather a coincidence, since $T_{1}$ is the same for both $\mathrm{PrOs}_{4} \mathrm{Sb}_{12}$ and $\mathrm{PrRu}_{4} \mathrm{Sb}_{12}$.

We then attempt to fit the low- $T$ nuclear magnetization with two $T_{1}$ components, namely,

$\frac{M_{0}-M(t)}{M_{0}}=$

$$
\sum_{i=S, L} a_{i}\left[0.0762 \exp \left(-\frac{3 t}{T_{1}^{i}}\right)+0.0146 \exp \left(-\frac{8.9 t}{T_{1}^{i}}\right)+0.9092 \exp \left(-\frac{17.3 t}{T_{1}^{i}}\right)\right],
$$

where $T_{1}^{S}$ means the short component due to fast relaxation, and $T_{1}^{L}$, the long component due to slow relaxation. The solid curve in Fig. 2 shows such fitting for $x=0.1$, with $a_{S}=0.8$ and $a_{L}=0.2$ that do not depend on temperature appreciably.

We first discuss the main component, $1 / T_{1}^{S}$, whose temperature dependence is shown in Fig. 3. Compared to the results for the end-member compounds, several trends can be seen. First, above $T \sim 100 \mathrm{~K}$, there is only one component of $T_{1}$ (namely, $T_{1}^{S}=T_{1}^{L}$ ), and the data 


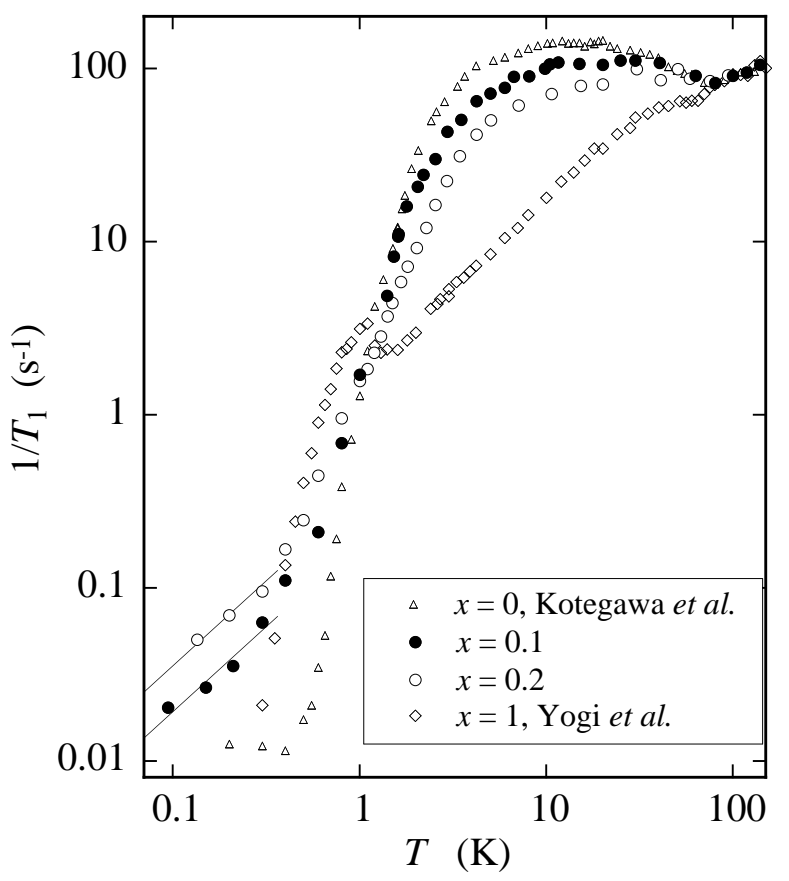

Fig. 3. Temperature dependence of $1 / T_{1}^{S}$ in $\operatorname{Pr}\left(\mathrm{Os}_{1-x} \mathrm{Ru}_{x}\right)_{4} \mathrm{Sb}_{12}(x=0.1,0.2)$. Data for $\mathrm{PrOs}_{4} \mathrm{Sb}_{12}{ }^{13}$ and $\mathrm{PrRu}_{4} \mathrm{Sb}_{12}$, which is a BCS superconductor, ${ }^{14}$ are also shown for comparison. The straight lines indicate the $T_{1} T=$ const relations.

for all $x$ merge into a same line. This suggests that the relaxation at high $T$ is not governed by the electronic state that is sensitive to the transition metal element, ${ }^{28}$ but due to other degree of freedom. Second, below $T \sim 100 \mathrm{~K}, 1 / T_{1}^{S}$ is close to that for pure $\mathrm{PrOs}_{4} \mathrm{Sb}_{12}$, but decreases as $x$ increases. Since the slow component $1 / T_{1}^{L}$ is very close to that for pure $\mathrm{PrRu}_{4} \mathrm{Sb}_{12}$, as will be shown later, we consider that $1 / T_{1}^{S}$ origenates from the Sb sites that are located far from Ru. Third, and most importantly, below $T \sim 1 \mathrm{~K}, 1 / T_{1}^{S}$ is proportional to $T$; the magnitude of $1 / T_{1}^{S} T$ increases with increasing $x$.

The $1 / T_{1}^{S} T=$ const. relation indicates that a finite DOS is induced by the impurity. $1 / T_{1}$ in the superconducting state may be expressed as

$$
\frac{T_{1}\left(T=T_{c}\right)}{T_{1}}=\frac{2}{k_{B} T_{c}} \int\left(N_{s}(E)^{2}+M_{s}(E)^{2}\right) f(E)(1-f(E)) d E,
$$

where $N_{s}(E)=N_{0} E /\left(E^{2}-\Delta^{2}\right)^{1 / 2}$ is the superconducting DOS with $\Delta$ being the superconducting gap, $M_{s}(E)=N_{0} \Delta /\left(E^{2}-\Delta^{2}\right)^{1 / 2}$ is the anomalous DOS due to the coherence factor, ${ }^{29} N_{0}$ is the DOS in the normal state and $f(E)$ is the Fermi function. In the $T=0$ limit, $1 / T_{1}$ is dominated by $N_{s}(0)^{2}$. The present results indicate that $N_{s}(0)$ is finite in the Ru-doped samples and increases with increasing $x$. This result cannot be accounted for by an $s$-wave gap. ${ }^{18-20}$ It is emphasized that only if there is a sign change in the gap function, namely, there are nodes in the gap function, can the impurity induce the finite DOS. ${ }^{20}$ Thus, our results provide strong evidence for the existence of nodes in the gap function. The seemingly expo- 


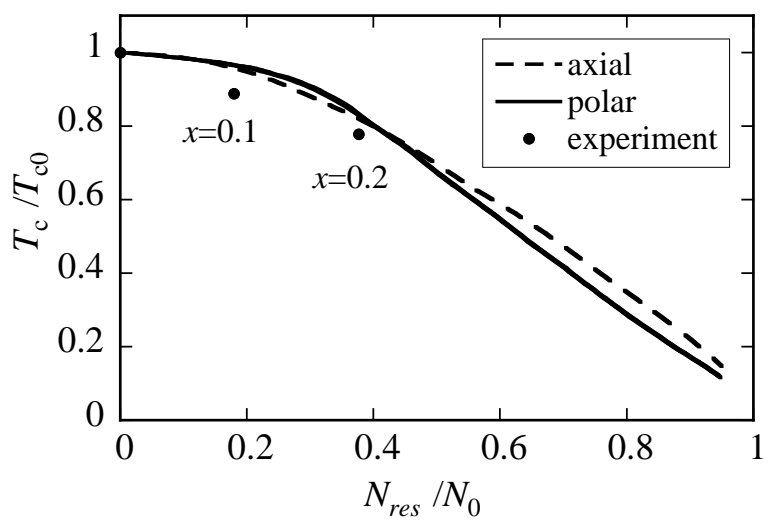

Fig. 4. Suppression of $T_{c}$ as a function of the residual DOS induced by the impurity, where $T_{c 0}$ is the transition temperature in the absence of impurity scattering. The curves indicate the calculated results obtained by Miyake. ${ }^{21,22}$

nential decrease in $1 / T_{1}$ with respect to $T$ in pure $\mathrm{PrOs}_{4} \mathrm{Sb}_{12}$ may be an effect obscured by the double superconducting transitions. ${ }^{30,31}$ Let us now estimate the impurity-induced $N_{s}(0)$, denoted as $N_{\text {res }}$ hereafter. The values of $N_{\text {res }}$ calculated from the relation of

$$
\frac{N_{\text {res }}}{N_{0}}=\sqrt{\frac{\left(T_{1}^{s} T\right)_{T_{c}}}{\left(T_{1}^{s} T\right)_{\text {low }-T}}}
$$

are shown in Fig. 4. The curves indicate the calculated results obtained by Miyake for the gaps with line-nodes (axial) and point-nodes (polar). ${ }^{21,22}$ The experimental results agree qualitatively with the theoretical results. That the experimental data fall below the theoretical curve is probably due to the depression of the pairing force by the substitution of $\mathrm{Ru}$, which is not included in the theoretical results. We will discuss this point later. Although we are unable to distinguish between line nodes and point nodes in Fig. 4, point nodes nonetheless seem more plausible, in view of the relatively weaker suppression of $T_{c}$ by the impurity with respect to $x .^{23}$

For completeness, we show the temperature dependence of $1 / T_{1}^{L}$ in Fig. 5 . Above $T_{c}, 1 / T_{1}^{L}$ shows a temperature variation very close to that of $\mathrm{PrRu}_{4} \mathrm{Sb}_{12}$. This $1 / T_{1}^{L}$ can be assigned to come from the $\mathrm{Sb}$ sites that are located close to $\mathrm{Ru}$. Below $T_{c}, 1 / T_{1}^{L}$ decreases rapidly, with no coherence peak.

Finally, we discuss the high-temperature behavior of $1 / T_{1}^{S}$. Figure 6 shows the temperature variation of the quantity $1 / T_{1} T$. Below $T \sim 6 \mathrm{~K}, 1 / T_{1} T$ decreases, leaving a peak at around $T \sim 6 \mathrm{~K}$. Such a decrease becomes mild as $x$ increases. Since the CEF gap $\Delta_{C E F}$ is small, Kotegawa et al. have analyzed the low- $T$ data by decomposing $1 / T_{1} T$ into two parts ${ }^{13}$ namely, the contribution due to the excitation to the $\Gamma_{4}^{(2)}$ state, and that due to heavy quasiparticles: $1 / T_{1} T=\alpha \times \exp \left(-\Delta_{C E F} / k_{B} T\right)+\beta \times 0.7 \mathrm{~s}^{-1} \mathrm{~K}^{-1}$, where $0.7 \mathrm{~s}^{-1} \mathrm{~K}^{-1}$ is the $1 / T_{1} T$ for $\mathrm{LaOs}_{4} \mathrm{Sb}_{12}$ and the parameter $\sqrt{\beta}$ represents the mass enhancement factor of the heavy quasiparticles. 


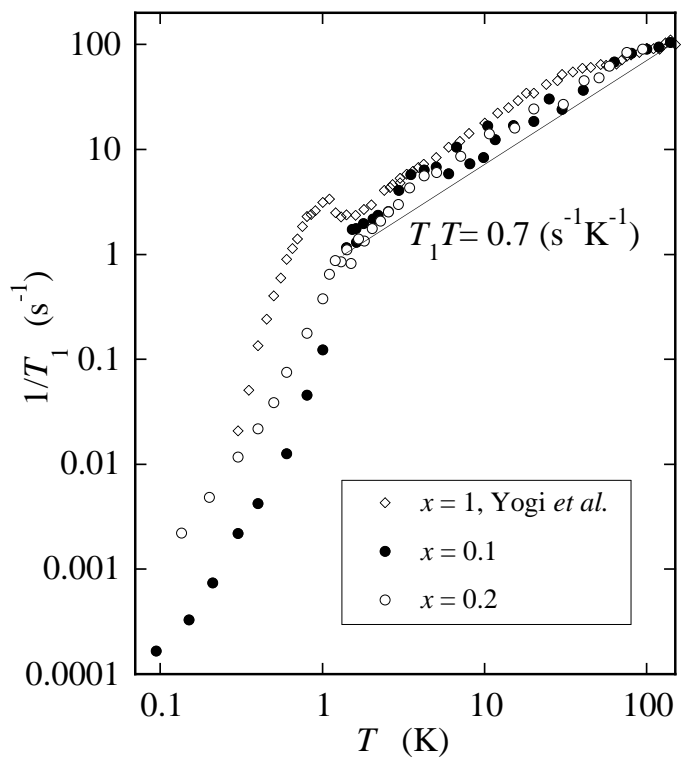

Fig. 5. Temperature dependence of the minor, slow relaxation component, $1 / T_{1}^{L}$. The straight line indicates the $1 / T_{1} T=0.7 \mathrm{~s}^{-1} \mathrm{~K}^{-1}$ relation found in $\mathrm{LaOs}_{4} \mathrm{Sb}_{12}$.

The application of the same analysis of Kotegawa et al. to the $x=0.1$ and 0.2 samples, as shown in the inset of Fig. 6 , shows that $\Delta_{C E F}$ increases with increasing $x$, while $\beta$ decreases with increasing $x$. The results are summarized in Table I, which are in good agreement with those reported by Frederick et al. who deduced $\Delta_{C E F}$ from the susceptibility and the mass enhancement factor from the specific heat coefficient $\gamma \cdot{ }^{23}$ As has been discussed by several authors, ${ }^{14,23}$ the increase in $\Delta_{C E F}$ is responsible, at least partly, for the decrease in $T_{c}$ in going from $\mathrm{PrOs}_{4} \mathrm{Sb}_{12}$ to $\mathrm{PrRu}_{4} \mathrm{Sb}_{12}$; thus, the discrepancy between the theoretical curve and the experimental data seen in Fig. 4 can be ascribed to the increase in $\Delta_{C E F}$.

In conclusion, we have studied the impurity effect on the superconductivity in the filled skutterudite heavy fermion superconductor $\mathrm{PrOs}_{4} \mathrm{Sb}_{12}$ using the NQR technique. We find that replacing Os with $\mathrm{Ru}$ brings about a finite density of states at the Fermi level, which increases with increasing $\mathrm{Ru}$ content. Our results provide strong evidence for the existence of nodes in the gap function.

\section{Acknowledgment}

We are grateful to Y. Kitaoka, H. Kotegawa, Yogi and Y. Imamura for helpful discussions and contribution. We would also like to thank K. Miyake for providing the unpublished calculated results shown in Fig. 4. This work was supported in part by a research grant from MEXT on the Priority Area "Skutterudites" (No. 15072204). 


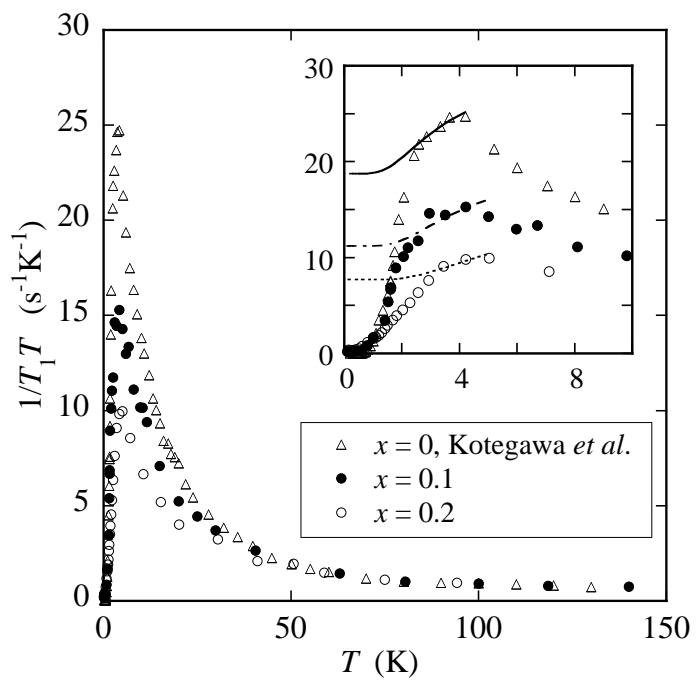

Fig. 6. Temperature dependence of the quantity $1 / T_{1}^{S} T$. The inset shows the enlarged part below $T=10 \mathrm{~K}$. The curves indicate the results of fitting $1 / T_{1}^{S} T=\alpha \times \exp \left(-\Delta_{C E F} / k_{B} T\right)+\beta \times 0.7$ $\mathrm{s}^{-1} \mathrm{~K}^{-1}$. For details, see the text. 


\section{References}

1) E. D. Bauer, N. A. Frederick, P.-C. Ho, V. S. Zapf and M. B. Maple: Phys. Rev. B 65 (2002) 100506.

2) M. B. Maple, P.-C. Ho, V. S. Zapf, N. A. Frederick, E. D. Bauer, W. M. Yuhasz, F. M. Woodward, and J. W. Lynn: J. Phys. Soc. Jpn. 71 (2002) Suppl. 23.

3) H. Sugawara, S. Osaki, S. R. Saha, Y. Aoki, H. Sato, Y. Inada, H. Shishido, R. Settai, Y. Onuki, H. Harima and K. Oikawa: Phys. Rev. B 66 (2002) 220504(R).

4) Y. Aoki, T. Namiki, S. Ohsaki, S. R. Saha, H. Sugawara and H. Sato: J. Phys. Soc. Jpn. 71 (2002) 2098.

5) K. Tenya, N. Oeschler, P. Gegenwart, F. Steglich, N. A. Frederick, E. D. Bauer and M. B. Maple: Acta Physica Polonica B 34 (2003) 995.

6) T. Tayama, T. Sakakibara, H. Sugawara, Y. Aoki and H. Sato: J. Phys. Soc. Jpn 72 (2003) 1516.

7) M. Kohgi, K. Iwasa, M. Nakajima, N. Metoki, S. Araki, N. Bernhoeft, J. M. Mignot, A. Gukasov, H. Sato, Y. Aoki and H. Sugawara: J. Phys. Soc. Jpn 72 (2003) 1002.

8) K. Kuwahara, K. Iwasa, M. Kohgi, K. Kaneko, S. Araki, N. Metoki, H. Sugawara, Y. Aoki and H. Sato: J. Phys. Soc. 73 (2004) 1438 .

9) E. A. Goremychkin, R. Osborn, E. D. Bauer, M. B. Maple, N. A. Frederick, W. M. Yuhasz, F. M. Woodward and J. W. Lynn: Phys. Rev. Lett. 93 (2004) 157003.

10) D. L. Cox: Phys. Rev. Lett. 59 (1987) 1240.

11) N. Takeda and M. Ishikawa: Physica B 259-261 (1999) 92.

12) T. D. Matsuda, K. Abe, F. Watanuki, H. Sugawara, Y. Aoki, H. Sato, Y. Inada, R. Settai and Y. Ōnuki: Physica B 312-313 (2002) 832.

13) H. Kotegawa, M. Yogi, Y. Imamura, Y. Kawasaki, G.-Q. Zheng, Y. Kitaoka, S. Ohsaki H. Sugawara, Y. Aoki and H. Sato: Phys. Rev. Lett. 90 (2003) 027001.

14) M. Yogi, H. Kotegawa, Y. Imamura, G.-Q. Zheng, Y. Kitaoka, H. Sugawara and H. Sato: Phys. Rev. B 67 (2003) 180501(R).

15) K. Asayama, Y. Kitaoka, G. - q. Zheng, K. Ishida and Y. Tokunaga: Annual Reports on NMR Spectroscopy (Academic Press, London, 2001) Vol. 44, p. 75.

16) D. E. Maclaughlin, J. E. Sonier, R. H. Heffner, O. O. Bernal, B. -L. Young, M. S. Rose, G. D. Morris, E. D. Bauer, T. D. Do and M. B. Maple: Phys. Rev. Lett. 89 (2002) 157001.

17) K. Izawa, Y. Nakajima, J. Goryo, Y. Matsuda, S. Osaki, H. Sugawara, H. Sato, P. Thalmeier and K. Maki: Phys. Rev. Lett. 90 (2003) 117001.

18) L. Yu: Acta Phys. Sinica 21 (1965) 75.

19) H. Shiba: Prog. Theor. Phys. 40 (1968) 435.

20) R. Fehrenbacher and M. R. Norman: Phys. Rev. B 50 (1994) 3495.

21) S. Schmitt-Rink, K. Miyake, and C. M. Varma: Phys. Rev. Lett. 57 (1986) 2575

22) K. Miyake: unpublished.

23) N. A. Frederick, T. D. Do, P.-C. Ho, N. P. Butch, V. S. Zapf and M. B. Maple: Phys. Rev. B 69 (2004) 024523.

24) E. E. M. Chia, M. B. Salamon, D. Vandervelde, D. Kikuchi, H. Sugawara and H. Sato: Condmat/0411395.

25) A. Abragam: The Principles of Nuclear Magnetism (Oxford Univ. Press, London, 1961).

26) G.-Q. Zheng, N. Yamaguchi, H. Kan, Y. Kitaoka, J. L. Sarrao, P. G. Pagliuso, N. O. Moreno and 
J. D. Thompson: Phys. Rev. B 70 (2004) 014511.

27) J. Chepin and J. H. Ross: J. Phys. Condens. Matt. 3 (1991) 8103.

28) H. Harima: unpublished.

29) D. E. Maclaughlin: Solid State Physics, eds. H. Ehrenreich et al. (Academic Press, New York, 1976) Vol. 31, p. 1.

30) R. Vollmer, A. Faißt, C. Pfleiderer, H. V. Löhneysen, E. D. Bauer, P.-C. Ho, V. Zapf and M. B. Maple: Phys. Rev. Lett. 90 (2003) 057001.

31) M.-A. Measson, D. Braithwaite, J. Flouquet, G. Seyfarth, J. P. Brison, E. Lhotel, C. Paulsen, H. Sugawara and H. Sato: Phys. Rev. B 70 (2004) 064516. 